\title{
The Possibility of Particles Forming from a Bose-Einstein Condensate, in an Intense Magnetic or Gravitational Field
}

\author{
Marius Arghirescu \\ State Office for Inventions and Trademarks, Bucharest, Romania \\ Email address: \\ arghirescu.marius@osim.ro,maris3a@yahoo.com \\ To cite this article: \\ Marius Arghirescu. The Possibility of Particles Forming from a Bose-Einstein Condensate, in an Intense Magnetic or Gravitational Field. \\ International Journal of High Energy Physics. Vol. 5, No. 1, 2018, pp. 55-62. doi: 10.11648/j.ijhep.20180501.16
}

Received: May 13, 2018; Accepted: May 29, 2018; Published: June 13, 2018

\begin{abstract}
In the paper - based on a previous work regarding the cold particles forming process as collapsed cold clusters of gammons- considered as pairs: $\gamma^{*}=\left(\mathrm{e}^{-} \mathrm{e}^{+}\right)$of axially coupled electrons with opposed charges, is analyzed the possibility of gammons pre-cluster forming from a Bose-Einstein condensate formed in the magnetic and in the gravitational field of a star. By known relations of a BEC forming, it is argued that- in the magnetic field of a star, the forming of a gammonic BEC with particles density $\mathrm{N}_{0}$ corresponding to those of a pre-cluster of gammons which may generates a particle-like stable cluster, may occurs- for a transition temperature $\mathrm{T}_{\mathrm{BE}} \approx 10^{3} \mathrm{~K}$, in a specific interval of field intensity and of temperature: $\mathrm{B}=\left(2.2 \times 10^{6} \div\right.$ $\left.8.3 \times 10^{7}\right) \mathrm{T}$ and $\mathrm{T}_{\mathrm{p}}=\left(4.8 \times 10^{-11} \div 1.8 \times 10^{-10}\right) \mathrm{K}$. The possible mechanism of the formed BEC transforming into pre-clusters of gammons which may become particle-like collapsed BEC, is a pearlitization mechanism, resulted as fragmentation of the formed BEC. It is argued that the particles forming from chiral quantum vacuum fluctuations is possible at $\mathrm{T} \rightarrow 0 \mathrm{~K}$, either by a vortexial, magnetic-like field corresponding to $\mathrm{B} \geq 10^{4} \mathrm{~T}$ or by already formed gammons, in a "step-by-step" process.
\end{abstract}

Keywords: Bose-Einstein Condensate, Bosonic Cluster, Elementary Particles, Magnetic Field Confining, Black Hole, Magnetar

\section{Introduction}

It is known that -according to the Superfluid vacuum theory (SVT, [1]), sometimes known as the „BEC vacuum theory", the fundamental physical vacuum is viewed as superfluid or as a Bose-Einstein condensate (BEC), with microscopic structure of fermion and antifermion pairs, describable by a macroscopic wave function, the visible matter appearing as excitations from this superfluid vacuum. In addition to single-particle excitations, the superfluid vacuum is capable of having real and virtual bound or quasibound composite excitations which are akin to bosons of integer spin which in particular are quanta of some interactions such as the electromagnetic interactions. However, the generation mechanism of real particles with non-null rest mass from virtual states of a quantum vacuum with low density, is not clear, even if the theory of the rest mass generation by the Higgs field gives an answer to this problem.

Also, the SVT not clarify some other problems such as the nature of force which generates virtual particle-like states, how the specific properties of each resulted particle/ subparticle are generated and how is explained the mass spectra of the generated particles, (for example, of those called "elementary particles", resulted from or by cosmic radiation).

In a theory developed by author in the book: "The cold Genesis of Matter and Fields" [2, 3] is argued the possibility of a cold genesis of elementary particles in a very strong magnetic field, comparable to those of a magnetar or gravistar, in accordance with a resulted quasi-crystalline model of quark and particle, resulted as Bose-Einstein condensate of $\mathrm{N}$ gammons, considered as pairs $\gamma^{*}\left(\mathrm{e}^{*_{+}}-\mathrm{e}^{*_{-}}\right)$of quasi-electrons with diminished mass $\mathrm{m}_{\mathrm{e}}{ }^{*}$, charge $\mathrm{e}^{*} \approx\left({ }^{2} / 3\right) \mathrm{e}$ and magnetic moment $\mu_{\mathrm{e}}{ }^{*}$, whose etherono-quantonic vortex of the magnetic moment: $\Gamma_{\mu}^{*}(\mathrm{r})=\Gamma_{\mathrm{A}}+\Gamma_{\mathrm{B}}$, is formed of sinergonic etherons $\left(\mathrm{m}_{\mathrm{s}} \approx 10^{-60} \mathrm{~kg}\right)$ - generating the magnetic potential $\mathrm{A}$ and of quantons $\left(\mathrm{m}_{\mathrm{h}}=\mathrm{h} / \mathrm{c}^{2}=7.37 \times 10^{-51} \mathrm{~kg}\right)-$ generating vortex-tubes that materializes the B-field lines of magnetic induction.

The used electron model is with the charge: e $=\mathrm{S}^{0} / \mathrm{k}_{1}$ contained by its surface $\mathrm{S}^{0}=4 \pi \mathrm{a}^{2}$ of radius: $\mathrm{a}=1.41 \mathrm{fm}-$ close to the value of the nucleon radius resulted from the 
expression of the nuclear volume: $r_{\mathrm{n}} \approx 1.25 \div 1.5 \mathrm{fm}$, and with an exponential variation of its density: $\rho_{e}=\rho_{e}^{0} \cdot e^{-r / \eta}$; with: $\rho_{e}^{0}$ $=22.24 \mathrm{~kg} / \mathrm{m}^{3}$ and $\eta=0.965 \mathrm{fm}$. It is argued that the electron may be formed at cold by an etherono-quantonic $\Gamma_{\mu}^{*}$-vortex of magnetic field vortex-tubes which materializes the B-field

$$
E_{s}(r)=k_{1} \cdot \rho_{e}(r) \cdot v_{\mathrm{c}}^{2}=\frac{1}{2} k_{1} \cdot \frac{\Delta p_{c}}{\Delta t} ; \quad \mathrm{q}_{\mathrm{s}}=\frac{4 \pi \cdot \mathrm{r}_{\mathrm{q}}^{2}}{\mathrm{k}_{1}} ;
$$

The resulted quark/particle model explains the nuclear force as an attraction of the nucleon's impenetrable volume $v_{i}$ in the field of $\mathrm{N}^{\mathrm{p}}=(2 \mathrm{~N}+1)$ - superposed vortices $\Gamma_{\mu}^{*}(\mathrm{r})$ of another nucleon, having an exponential variation of quanta impulse density: $p_{\mu}=\rho_{\mu} c$, according to equation:

$$
\mathrm{V}_{\mathrm{n}}(\mathrm{r})=v_{\mathrm{i}} \mathrm{P}_{\mathrm{n}}=\mathrm{V}_{\mathrm{n}}{ }^{0} \cdot \mathrm{e}^{-\mathrm{r} / \eta^{*}} ; \mathrm{V}_{\mathrm{n}}{ }^{0}=\mathrm{v}_{\mathrm{i}} \mathrm{P}_{\mathrm{n}}{ }^{0 ;} \mathrm{P}_{\mathrm{n}}(\mathrm{r})=(1 / 2) \rho_{\mathrm{n}}(\mathrm{r}) \cdot \mathrm{c}^{2}
$$

with: $v_{\mathrm{i}}(0.6 \mathrm{fm}) \approx 0.9 \mathrm{fm}^{3}$ - the impenetrable quantum volume of the nucleon; $\rho_{n}{ }^{0} \approx N^{p} \cdot \rho_{e}{ }^{0}$, and with $\eta^{*}=0.755 \mathrm{fm}$, resulted by the condition: $\rho_{\mu}\left(a, \mathrm{e}^{*}\right) \approx \rho_{e}\left(a, \mathrm{e}^{*}\right)=\left({ }^{2} / 3\right) \rho_{e}(a, \mathrm{e}) \sim \mathrm{e}^{*}=$ $(2 / 3)$ e, [2-4].

The proton results in CBT by a neutral part with an attached electron (as in the Anderson's model [5]).

The virtual radius: $r_{\mu}{ }^{n}$ of the proton's magnetic moment, $\mu_{\mathrm{p}}$, results - in the theory, by a degenerate Compton radius $r_{\lambda}$ $=\lambda / 2 \pi=\hbar / m_{p} c$ of the attached positron, which decreased when the protonic positron was included in the $\mathrm{N}^{\mathrm{p}}$ cluster volume, $\left(\mathrm{N}^{\mathrm{p}}\right.$-the number of quasielectrons of the particle), from the value: $r_{\mu}^{e}=3.86 \times 10^{-13} \mathrm{~m}$, to the value: $r_{i}=r_{\mu}{ }^{p}=$ $0,59 \mathrm{fm}$, as a consequence of the increasing of the impenetrable quantum volume mean density in which is included the protonic positron centrol: $\mathrm{m}_{0}$, from the value: $\overline{\rho_{\mathrm{e}}}$ to the value: $\overline{\rho_{n}} \cong f_{d} \cdot N^{p} \cdot \rho_{e}$.

This phenomenon may explain the neutron according to a "dynamid" model, with a degenerate electron with degenerate magnetic moment: $\mu_{\mathrm{e}}^{\mathrm{s}}=-4.597 \mu_{\mathrm{N}}$ rotated inside the quantum volume of a proton by the etherono-quantonic vortex $\Gamma\left(\mu_{\mathrm{p}}\right)$ of its magnetic moment $\mu_{\mathrm{p}}$, with a speed $\mathrm{v}_{\mathrm{e}} \approx$ $1.7 \times 10^{-2} \mathrm{c}$, to an orbital with a radius: $\mathrm{r}_{\mathrm{e}}{ }^{*} \approx 1.283 \mathrm{fm}[6]$ under dynamic equilibrium of forces on tangent and radial directions. The difference between the neutron' mass and the proton' mass: $\Delta_{\mathrm{n}} \approx 2.6 \mathrm{~m}_{\mathrm{e}}$, is given by a "weson" $\mathrm{w}^{\text {- }}$ composed of the attached negatron and a linking gammon: $\sigma\left(\mathrm{e}^{*_{+}}-\mathrm{e}^{*_{-}}\right)$which is transformed into an electronic lines of a magnetaric field $\mathrm{B} \rightarrow 10^{12} \mathrm{~T}$ formed around an electronic superdense kernel (centroid). approximated as cylindrical, with $\mathrm{r}_{0} \approx 10^{-18} \mathrm{~m}$. The electric field intensity $\mathrm{E}$, the electric charge $\mathrm{q}$ and the magnetic induction $\mathrm{B}$ the results in the form:

$\mathrm{B}=\mathrm{k}_{1} \cdot \rho_{\mu}(r) \cdot \mathrm{v}_{\mathrm{c}} ;\left(k_{1}=\frac{4 \pi \cdot a^{2}}{e}=1.56 \times 10^{-10} \frac{\mathrm{m}^{2}}{C} ; \mathrm{v}_{\mathrm{c}} \approx c\right)$

antineutrino given as pair of electronic centroids (with null spin, identical with the electronic neutrino, resulted as Majorana particle), by the loss of the quantum volume of quasielectrons, the quasielectron mass resulting of value: $\mathrm{m}_{\mathrm{e}}{ }^{*}$ $=\left(\Delta_{\mathrm{n}}-1\right) / 2 \approx 0.81 \mathrm{~m}_{\mathrm{e},}[2-4,6]$.

${ }^{0} \mathrm{n}_{\mathrm{e}}=\left(\mathrm{N}^{\mathrm{p}}+\mathrm{w}^{-}\right)=\left(\mathrm{N}^{\mathrm{p}}+\mathrm{e}^{-}+\sigma\right) \rightarrow{ }^{1} \mathrm{p}_{\mathrm{r}}+\mathrm{e}^{-}+\overline{\mathrm{v}}_{\mathrm{e}}+\epsilon_{\sigma}(889 \mathrm{keV}) ;$

The model may explain also the reaction of $\mathrm{K}$-electron capture: $p_{r}+e^{+} \rightarrow n_{e}+v_{e}$, by the conclusion that the electronic neutrino results- in this reaction, by the coupling of the electron's superdense centroid with the centroid of the protonic positron having degenerate magnetic moment, with the loss of the quantum volume.

The theory, which predicted the existence of a preon $z^{0} \approx$ $34 \mathrm{~m}_{\mathrm{e}}$, experimentally evidenced in 2015 but considered as $\mathrm{X}$ - boson of a fifth force, of leptons to quark binding, argues a preonic model of quarks whose stability was explained by a quasi-crystalline model of $z^{0}$-preon and of the quark core.

By CGT was argued the fact that- in a cold genesis model, the preon $z^{0}$ may results as cluster of $n=42$ degenerate electrons with the mass: $\mathrm{m}_{\mathrm{e}}^{*} \approx 0.81 \mathrm{~m}_{\mathrm{e}}$ and with the superdense kernels (centroids) vortexially confined in a volume of radius $r_{z}<0.2 \mathrm{fm}$, the $\mathrm{z}^{0}$ cluster being given as a pair of quarcins: $\mathrm{c}^{* \pm}=21 \mathrm{~m}_{\mathrm{e}}{ }^{*}$, with hexagonal symmetry $(7 \mathrm{x} 3=21$ quasielectrons).

The resulted sub-structure of the fundamental elementary particles, considered as formed "at cold", by quarks with effective mass (giving the particle's mass by the sum rule) and fractional electric charge $\mathrm{q}^{*}=(+2 / 3 \mathrm{e} ;-1 / 3 \mathrm{e})$, formed as preonic clusters, is given by the preon $z^{0}$ considering also the existence of the zerons: $z_{1}=3 z^{0}=102 m_{e} ; z_{2}=4 z^{0}=136 m_{e}$; $\mathrm{z}_{\pi}=\left(\mathrm{z}_{1}+\mathrm{z}_{2}\right)=7 \mathrm{z}^{0}=238 \mathrm{~m}_{\mathrm{e}}$, with the following sub-structures $[2-4,6]$ :

Table 1. Elementary particles: (theoretic mass) / (experimentally determined mass).

$\begin{array}{ll}\text { Basic quarks: } \mathrm{m}_{1}=\left(\mathrm{z}_{2^{-}} \mathrm{m}_{\mathrm{e}}{ }^{*}\right)=135.2 \mathrm{~m}_{\mathrm{e}}, & \mathrm{m}_{2}^{-}=\mathrm{m}_{1}+\mathrm{e}^{-}+\sigma_{\mathrm{e}}=137,8 \mathrm{~m}_{\mathrm{e}} ; \mathrm{m}_{2}^{-} \rightarrow \mathrm{m}_{1}+\mathrm{e}^{-}+\overline{\mathrm{v}}_{\mathrm{e}} ;\left(\sigma_{\mathrm{e}}=\left(\mathrm{e}^{+*}+\mathrm{e}^{-*}\right) \rightarrow \bar{v}_{\mathrm{e}}\right) \\ \text { Derived quarks: } \mathrm{p}^{+}\left(\mathrm{n}^{-}\right)=\mathrm{m}_{1}\left(\mathrm{~m}_{2}\right)+2 \mathrm{z}_{\pi} & \mathrm{n}^{-}=\mathrm{p}^{+}+\mathrm{e}^{-}+\sigma_{\mathrm{e}} \rightarrow \mathrm{p}^{+}+\mathrm{e}^{-}+\overline{\mathrm{v}_{\mathrm{e}}} ; \lambda=\mathrm{n}^{-}(\mathrm{p})+\mathrm{z}_{\pi} ; \mathrm{s}=\lambda+\mathrm{z}_{2} ; \mathrm{v}^{-}=\lambda \lambda^{-}+2 \mathrm{z}_{2} \\ \text { Mesons: }(\mathrm{q}-\overline{\mathrm{q}}) & \text { Baryons: }(\mathrm{q}-\mathrm{q}-\mathrm{q}) \\ \mu^{-}=2 \mathrm{Z}_{1}+\mathrm{e}^{-}=205 \mathrm{~m}_{\mathrm{e}} / \mu^{+}=206.7 \mathrm{~m}_{\mathrm{e}} & -\mathrm{p}_{\mathrm{r}}=2 \mathrm{p}+\mathrm{n}=1836.2 \mathrm{~m}_{\mathrm{e}} ; \mathrm{n}_{\mathrm{e}}=2 \mathrm{n}+\mathrm{p}=1838.8 \mathrm{~m}_{\mathrm{e}} ; / \mathrm{p}_{\mathrm{r}}^{+}, \mathrm{n}_{\mathrm{e}}=1836.1 ; 1838.7 \mathrm{~m}_{\mathrm{e}} ; \\ \pi^{\mathrm{o}}=\mathrm{m}_{1}+\overline{\mathrm{m}}_{1}=270.4 \mathrm{~m}_{\mathrm{e}} ; \pi^{0}=264.2 \mathrm{~m}_{\mathrm{e}} & -\Lambda^{\mathrm{o}}=\mathrm{s}+\mathrm{n}+\mathrm{p}=2212.8 \mathrm{~m}_{\mathrm{e}} ; / \Lambda^{0}=2182.7 \mathrm{~m}_{\mathrm{e}} \\ \pi^{+}=\mathrm{m}_{1}+\overline{\mathrm{m}}_{2}=273 \mathrm{~m}_{\mathrm{e}} ; / \pi^{+}=273.2 \mathrm{~m}_{\mathrm{e}} & -\Delta^{(++;+; 0 ;-)}=\mathrm{s}^{ \pm}+\lambda^{ \pm}+\mathrm{p}^{+}\left(\mathrm{n}^{-}\right)=2445.6 ; 2453.4 \mathrm{~m}_{\mathrm{e}} ; / \Delta^{ \pm 0}=2411 \pm 4 \mathrm{~m}_{\mathrm{e}} \\ \mathrm{K}^{+}=\mathrm{m}_{1}+\bar{\lambda}=987 \mathrm{~m}_{\mathrm{e}} ; \mathrm{K}^{+}=966.3 \mathrm{~m}_{\mathrm{e}} & -\Sigma^{+}=\mathrm{v}+2 \mathrm{p}=2346.2 \mathrm{~m}_{\mathrm{e}} ; \Sigma^{-}=\mathrm{v}+2 \mathrm{n}=2351.4 \mathrm{~m}_{\mathrm{e}} ; / \Sigma^{+}, \Sigma^{-}=2327 ; 2342.6 \mathrm{~m}_{\mathrm{e}} \\ \mathrm{K}^{\mathrm{o}}=\mathrm{m}_{2}+\bar{\lambda}=989.6 \mathrm{~m}_{\mathrm{e}} ; / \mathrm{K}^{\mathrm{o}}=974.5 \mathrm{~m}_{\mathrm{e}} & -\Sigma^{\mathrm{o}}=\mathrm{v}+\mathrm{n}+\mathrm{p}=2348.8 \mathrm{~m}_{\mathrm{e}} / \Sigma^{0}=2333 \mathrm{~m}_{\mathrm{e}} ; \\ \eta^{\mathrm{o}}=\mathrm{m}_{2}+\overline{\mathrm{s}}=1125.6 \mathrm{~m}_{\mathrm{e}} ; \eta^{0}=1073 \mathrm{~m}_{\mathrm{e}} ; & -\Xi^{\mathrm{o}}=2 \mathrm{~s}+\mathrm{p}=2586.8 \mathrm{~m}_{\mathrm{e}} ; \Xi^{-}=2 \mathrm{~s}+\mathrm{n}=2589.4 \mathrm{~m}_{\mathrm{e}} ; / \Xi^{0}, \Xi^{-}=2572 ; 2587.7 \mathrm{~m}_{\mathrm{e}} ; \\ & -\Omega^{-}=3 \mathrm{v}=3371.4 \mathrm{~m}_{\mathrm{e}} ; / \Omega^{-}=3278 \mathrm{~m}_{\mathrm{e}} .\end{array}$

The classical, pre-quantum mechanism of the preonic cold quarks forming and of dark matter bosons forming was explained in a relative recent paper of the author [7] by the cold genesis of two preonic bosons with hexagonal 
symmetry: $z_{\pi}=7 z^{0} ; z_{2}=4 z^{0}$, by the magnetic interaction between constituents, as a mechanism with the next steps:

a) $\mathrm{z}^{0^{*} /} \mathrm{z}^{0}$-pre-cluster/cluster forming, with the aid of magnetic confinement; b) $z_{2}{ }^{*} / \mathrm{z}_{2^{-}}$and $\mathrm{z}_{\pi}{ }^{*} / \mathrm{z}_{\pi^{-}}$precluster/cluster forming; c) $\left(\mathrm{q}^{ \pm /} \mathrm{q}^{0}\right)$ - quark or pseudo-quark precluster/cluster forming; d) pre-cluster of quarks or pseudoquarks forming; e) elementary particle/dark boson forming.

The total collapsing is impeded- according to the model, by a repulsive force of a scalar field generated by remanent thermal vibrations of the electronic centroids (kernels) which determines a local increasing of the quantum static pressure.

The formed structures corresponds- according to the model, to an organized (quasi-crystallin) collapsed BoseEinstein condensate [7], with quasi-crystallin kernel given by an arrangement with hexagonal symmetry of the electronic centroids, having- in the model, a barel-like form, with a diameter of $10^{-18} \mathrm{~m}[2-4]$.

The possibility of Bose-Einstein condensate forming in a magnetic field and in a gravitational field was studied in some reference papers $[8,9]$.

The collapsing of a BEC, corresponding to a sharply density increasing of the formed condensate, was obtained experimentally with $\sim 15000$ atoms of ${ }^{85} \mathrm{Rb}$ cooled in a magnetic trap at $\sim 3 \mathrm{nK}$ with $\mathrm{B} \approx 162 \mathrm{Gs}$ [10], being observed a remnant condensate as fraction of the initial condensate and a missing atoms part.

The stability of the remnant condensate that was observed during the collapse of attractive BECs was explained by the existence of an absolute stable minimum that appears only if the energy is nonlocal, as consequence of the condensate's transition which occurs when the scattering length ' $a$ ' from the Gross-Pitaevskii equation is tuned from zero scattering length to a negative scattering length, determined by the magnetic field, as a linear function of $1 /\left(\mathrm{B}-\mathrm{B}_{0}\right)$, where $\mathrm{B}_{0}$ is the Feshback resonance position [11].

It was shown also that the transition temperature of a BEC formed in a magnetic field, decreases with the strength of the B-field, according to the relation: $(\overline{\mathrm{B}})=T_{0}\left[1-\left({ }^{2} / 5\right) \cdot R(\overline{\mathrm{B}})\right]$, with $R(\overline{\mathrm{B}}) \sim 1+(\overline{\mathrm{B}})^{2}$.

It was considered theoretically also the possibility to describe the dark matter as a non-relativistic, Newtonian Bose-Einstein gravitational condensate, [12] but at the atomic level and the possibility to consider a black hole as a BoseEinstein condensate of gravitons [13].

In the present paper we analyze the possibility of gammons pre-clusters forming, specific to cold formed quarks or pseudo-quarks (corresponding to neutral bosons), as Bose-Einstein condensate produced in the magnetic and the gravitational field of a star which ensures at its surface the condition: $\mathrm{T}<\mathrm{T}_{\mathrm{BE}}$.

\section{The Bose-Einstein Condensate of Gammons in a Magnetic Field}

According to a pre-quantum analyze of the forming process of a collapsed cluster of gammons considered as gammonic pairs: $\gamma^{*}=\left(\mathrm{e}^{-} \mathrm{e}^{+}\right)$of axially coupled electrons with opposed charges [7], it resulted that -under a specific BoseEinstein condensate forming temperature, $\mathrm{T}_{\mathrm{BE}}$, the magnetic interaction between the gammons is enough for the gammons pre-cluster forming and self-confining, with the forming of a stable particle-like cluster, if the external magnetic field $B$ not exceed a critical value $B_{c}$ given by the condition that the $\mathrm{V}_{\mathrm{B}}\left(\mathrm{B}-\gamma^{*}\right)$ potential to be lower than the $\mathrm{V}_{\gamma}\left(\gamma^{*}-\gamma^{*}\right)$ potential: $\mathrm{V}_{\mathrm{B}}=\mu_{\mathrm{e}} \cdot \mathrm{B} \leq \mathrm{V}_{\gamma}(\mathrm{r})=\mu_{0} \mu_{\mathrm{e}}^{2} / 2 \pi \mathrm{r}^{3}$.

The gammonic pairs may results -in the magnetic field of a neutronic star, from nuclear $\gamma$-emissions resulted particularly by K-electron capture: $\mathrm{p}_{\mathrm{r}}+\mathrm{e}^{+} \rightarrow \mathrm{n}_{\mathrm{e}}+\gamma$ and by combinations between electrons resulted from attracted atoms and positrons resulted as $\beta^{+}$-radiation emitted by protons transformed into neutrons: $\mathrm{p}_{\mathrm{r}} \rightarrow \mathrm{n}_{\mathrm{e}}+\mathrm{e}^{+}+\mathrm{v}$, which are trapped in the star's magnetic field, in which has the Hamiltonian:

$$
H=\frac{(p-\mathrm{e} \cdot \bar{A})^{2}}{2 m}-\frac{e \cdot \hbar}{2 m} \bar{\sigma} \cdot \bar{B}=\frac{p_{\lambda}^{2}}{2 m}-\bar{\mu} \cdot \bar{B}
$$

with $\mathrm{A}, \mathrm{B}, \mathrm{p}_{\lambda}$-the magnetic potential, the magnetic induction and respective-the canonic impulse.

Is of particular interest to know which category of stars may offer conditions for particles cold genesis.

It is known that the quantum nature of a cold gas is evident when the distance between particles is smaller than the thermal wavelength, i.e: $l_{i}=\mathrm{N}^{-1 / 3} \leq \lambda_{t}=h / p_{k},\left(p_{k}=\sqrt{ }\left(2 m E_{k}\right)\right)$. In a magnetic $B$-field, the energy of a charged particle, $\mathrm{m}$, is: $E_{B}=p^{2} / 2 m+\mu_{e} \cdot B ;\left(\mu_{e}=e \hbar / 2 m \cdot c\right)$.

Because that in CGT the gammons have a magnetic moment $\mu_{\gamma}$ given by the axially coupling of negatron and positron, according to the similitude principle, we may use for the proposed study a relation of the $\mathrm{T}_{\mathrm{BE}}$ as those deduced by Rojas and Villegas for the non-relativistic case $\left(\mathrm{E}_{\mathrm{k}}=\right.$ $\pi \mathrm{k}_{\mathrm{B}} \mathrm{T}$ ) of charged particles [8], of the form:

$$
\begin{gathered}
\frac{L}{\lambda_{t}} \cong v \cdot N ; \quad \Rightarrow \quad T_{B}=\frac{4 \pi^{2} \hbar^{3} c}{e B m \cdot k_{B} L} N=\frac{2 \pi^{2} \hbar^{4}}{\mu_{1} m^{2} k_{B} L} N ; \quad \mu_{1}=\mu_{\mathrm{e}} \cdot B \gg k_{B} T \\
\frac{\mathrm{N}_{0}}{\mathrm{~N}}=\frac{\lambda_{t}}{L \cdot \sqrt{\mu_{1}}} ; \quad \mathrm{N}=\frac{\sqrt{\pi}}{v \sqrt{\mu_{1}}} ; \quad v=\frac{\lambda_{\mathrm{t}} h \cdot c}{e \cdot B}=\frac{\lambda_{t} \hbar^{2} \pi}{\mu_{1} m} ; \quad \lambda_{t}=\frac{\mathrm{h}}{\sqrt{2 \pi \mathrm{m} \cdot k_{B} \mathrm{~T}}}
\end{gathered}
$$

in which: $T_{B}$ - the temperature necessary for having a macroscopic fraction of the total density of charged particles in the ground state; L - the dimension of the ground state system parallel to the external magnetic B- field; $N ; N_{0}$ - the density of $\mathrm{m}$ - particles outside and in the B-E condensate; $\lambda_{t}$ the thermal wavelength; $v=\lambda_{\mathrm{t}} \mathrm{hc} / \mathrm{eB}$ - the elementary cell and 
$\mathrm{eBh} / 2 \mathrm{~m} \cdot \mathrm{c}=\mu_{1}-$ the effective chemical potential, corresponding to a magnetic potential: $\mu_{1}=\mu_{\mathrm{e}} \cdot \mathrm{B}$.

For $\mathrm{T}<<\mathrm{T}_{\mathrm{B}}$ we have almost all the particles in the ground state $\mathrm{n}=0$ and a true BEC exists.

From eqns. (4a) and (4b) it results that:

$$
\mathrm{N}=\frac{\sqrt{\pi}}{v \sqrt{\mu_{1}}}=\sqrt{\frac{\mu_{1}}{\pi}} \frac{m}{\lambda_{l} \hbar^{2}}=\frac{L \sqrt{\mu_{1}}}{\lambda_{t}} N_{0} \quad ; \quad \Rightarrow \mathrm{L}=\frac{\mathrm{m}}{\mathrm{N}_{0} \hbar^{2} \sqrt{\pi}} ;
$$

Considering-according to CGT [7], that the pre-cluster corresponding to the un-collapsed Bose-Einstein condensate of gammons $\left(\mathrm{m}=\mathrm{m}_{\gamma} \approx 2 \mathrm{~m}_{\mathrm{e}}=1.82 \times 10^{-30} \mathrm{~kg}\right)$ has the distance between the degenerate electrons, of meta-stable equilibrium, of value: $\mathrm{a}=1.41 \mathrm{fm}$, it results from eq. (6) that: $\mathrm{N}_{0} \approx 1 / \mathrm{a}^{3}=$ $3.57 \times 10^{44}$ and: $\mathrm{L} \approx 2.5 \times 10^{-7} \mathrm{~m}$.

The pre-cluster of a cold formed quark ( $\sim 377$ degenerate gammons) corresponds- in consequence, to a fragmentation part, of radius $r_{p}=a \cdot(377)^{-1 / 3} \approx 7.2 \mathrm{a}=10.2 \mathrm{fm}$, resulted as consequence of a 'pearlitization' phenomenon generated by the decreasing of the inter-distance $l_{\mathrm{i}}=\mathrm{N}^{-1 / 3}<\lambda_{\mathrm{t}}$, the quasicrystallin form of the pre-cluster and thereafter- of the particle-like collapsed cluster, resulting by magnetic interaction between 'residual' magnetic moments of the gammonic bosons [7].

In the absence of a magnetic field and a gravitic field, supposing that it exists a concentration of gammons $\mathrm{N} \approx$ $10^{24}$,for example, it results-according to the specific relation of BEC transition temperature:

$$
T_{B E}(\mathrm{~B}=0)=3.3125 \cdot \frac{\hbar^{2}}{m \cdot k_{B}} N^{\frac{2}{3}}
$$

that the temperature of transition to a $\mathrm{BEC}$ state is of the order: $\mathrm{T}_{\mathrm{BE}}^{1}=1464 \mathrm{~K}$.

Because that- according to eqn. (4), the external B-field decreases the value of $T_{\mathrm{BE}}$, we may choose a test temperature $\mathrm{T}_{\mathrm{B}} \approx 10^{3} \mathrm{~K}$. Also, considering a degenerate charge of quasielectrons forming the gammonic pair equal to those of up-quark [2-4]: $\mathrm{e}^{*} \approx(2 / 3)$ e and a magnetic moment of the gammon: $\mu_{\gamma} \approx\left(\mathrm{m}_{\mathrm{e}} / \mathrm{m}_{\gamma}\right) \cdot\left({ }^{2} / 3\right) \mu_{\mathrm{BP}} \approx 3 \times 10^{-24} \mathrm{~J} / \mathrm{T},\left(\mu_{\mathrm{BP}}-\right.$ the Procopiu-Bohr magneton: $9.28 \times 10^{-24} \mathrm{~J} / \mathrm{T}$ ), resulted from the un-canceled axially coupled magnetic moments of the $\mathrm{e}^{* \pm}$ charges, it results from eqns. (3) and (4) that:

$$
\mathrm{N}=\frac{L \sqrt{\mu_{1}}}{\lambda_{t}} N_{0}=\frac{\mu_{1} m_{\gamma}^{2} k_{B} T_{B} L}{2 \pi^{2} \hbar^{4}} ; \Rightarrow \lambda \sqrt{\mu_{1}}=h \sqrt{\frac{\mu_{1}}{2 \pi \cdot m_{\gamma} k_{B} T_{p}}}=\frac{2 \pi^{2} \hbar^{4}}{m_{\gamma}^{2} k_{B} T_{B}} \mathrm{~N}_{0} ; \sqrt{\frac{\mu_{1}}{\mathrm{~T}_{\mathrm{p}}}}=\frac{\pi \cdot \hbar^{3} N_{0}}{T_{B}} \sqrt{\frac{2 \pi}{m^{3} k_{B}}}
$$

With $\mathrm{T}_{\mathrm{B}} \approx 10^{3}$, it results that: $\lambda_{\mathrm{t}} \sqrt{ } \mu_{1}=1.9 \times 10^{-11}$ and $\sqrt{ }\left(\mathrm{T}_{\mathrm{p}} / \mu_{1}\right)=2.7 \mathrm{x} \mathrm{T}_{\mathrm{B}}$, with $\mathrm{T}_{\mathrm{p}}$ - the temperature to which the precluster $\mathrm{P}^{\mathrm{c}}$ of gammonic particles density $\mathrm{N}_{0}$ is formed. Choosing some values for $T_{B}$ and $B$, will results the corresponding values for $\mu_{1}=\mu_{\gamma} \cdot \mathrm{B}$ and for $T_{p}$. For example, for $\mathrm{T}_{\mathrm{B}}=10^{3} \mathrm{~K}$ and $\mathrm{B}=10^{6} \mathrm{~T}$, it results that $\mathrm{T}_{\mathrm{p}} \approx 2.2 \times 10^{-11} \mathrm{~K}$, $\lambda_{\mathrm{t}}=1.12 \times 10^{-2} \mathrm{~m}$ and $\mathrm{N}=1.38 \times 10^{31}$.

The previous conclusion of the gammon's magnetic moment value is based on the CGT's conclusion that the etherono-quantonic vortex $\Gamma_{\mu}=2 \pi r_{\lambda} \mathrm{c}$ induced by the chirality of the electron's superdense kernel (centroid) is the cause of the electron's charge forming and not inverse.

Linde [14] suggested that very high values of the magnetic field, exceeding $10^{11} \mathrm{~T}$, may generate the condensation of $\mathrm{W}^{ \pm}$ bosons resulted from the electro-weak interaction theory.

In our case, a magnetic field $\mathrm{B} \approx 10^{11} \mathrm{~T}$ gives- with $\mathrm{T}_{\mathrm{B}}=$ $10^{3} \mathrm{~K}$, a value $\mathrm{T}_{\mathrm{p}} \approx 2.2 \times 10^{-6} \mathrm{~K}$. But- according to CGT, for the $\mathrm{P}^{\mathrm{c}}$-pre-cluster collapsing the potential $\mu_{1}$ must be lower than the potential of magnetic interaction between gammons at a rougly approximate distance $\mathrm{d}_{\lambda}=\lambda / 2 \pi$ with $\lambda=\mathrm{h} / \mathrm{m}_{\gamma} \mathrm{c}=$ $1.93 \times 10^{-13} \mathrm{~m}: \mathrm{V}_{\mu}=\mu_{0} / 2 \pi\left(\mu_{\gamma}{ }^{2} / \mathrm{d}_{\lambda}{ }^{2}\right)=2.5 \times 10^{-16} \mathrm{~J}$, resulting thatfor the pre-cluster's forming, it is necessary a B -field lower than a critical value: $B_{c}=8.3 \times 10^{7} \mathrm{~T}$ or a supplementary force that may compensate the repulsion between the gammonic magnetic moments oriented parallel with the field $\mathrm{B}>\mathrm{B}_{\mathrm{c}}$.

The inferior limit of the B-field is given by the conclusion that the quantum vacuum temperature is given by quantons, $\left(\mathrm{m}_{\mathrm{h}}=7.37 \times 10^{-51} \mathrm{~kg}\right)$ according to the relation: $\mathrm{k}_{\mathrm{B}} \mathrm{T}_{\mathrm{h}} \approx \mathrm{m}_{\mathrm{h}} \mathrm{c}^{2}$ resulting that $\mathrm{T}_{\mathrm{h}} \approx 4.8 \times 10^{-11} \mathrm{~K}$, which corresponds by eqn. (8) and by $\mathrm{T}_{\mathrm{B}}=10^{3} \mathrm{~K}$, to $\mathrm{B}_{1}=2.2 \times 10^{6} \mathrm{~T}$ and which is comparable with the Hawking's temperature at which the surface of a massive "black hole" of mass $\mathrm{M} \geq 10^{5}$ solar masses may radiate (may emits Hawking's radiation).

We may conclude also that the dynamic pressure of the quantonic vortex-tubes which materializes the magnetic induction field lines - according to CGT, may decrease the temperature $\mathrm{T}_{\mathrm{h}}$ of the quantum vacuum.

Also, if we choose a lower transition temperature $T_{B}$, for example: $T_{B}=10^{2} \mathrm{~K}$, by eqn. (8) and with $\mathrm{B}=\mathrm{B}_{1}=2.2 \times 10^{6} \mathrm{~T}$ (or with $\mathrm{T}_{\mathrm{B}}=10^{3} \mathrm{~K}$ but with a lower $\mathrm{B}$-field), will results a temperature of the $\mathrm{P}^{\mathrm{c}}$-pre-cluster forming: $\mathrm{T}_{\mathrm{p}}=4.8 \times 10^{-13} \mathrm{~K}$ lower than the considered limit $\mathrm{T}_{\mathrm{h}}$.

So, maintaining the test temperature $\mathrm{T}_{\mathrm{B}}=10^{3} \mathrm{~K}$, it results that we have an interval: $\mathrm{B}=\left(2.2 \times 10^{6} \div 8.3 \times 10^{7}\right) \mathrm{T}$ in which is fulfilled the condition: $\mathrm{T}_{\mathrm{p}} \geq \mathrm{T}_{\mathrm{h}}$ and which corresponds by eqn. (8) to: $\mathrm{T}_{\mathrm{p}}=\left(4.8 \times 10^{-11} \div 1.8 \times 10^{-10}\right) \mathrm{K}$.

The gammonic particles concentration $\mathrm{N}$ corresponding to the values: $\mathrm{T}_{\mathrm{B}}=10^{3} \mathrm{~K} ; \mathrm{B}=\left(2.2 \times 10^{6} \div 8.3 \times 10^{7}\right) \mathrm{T}$, results- from eqn. (8), of value:

$\mathrm{N}=\mathrm{N}_{0} \mathrm{~L} \cdot \mu_{1} / \lambda_{\mathrm{t}} \sqrt{ } \mu_{1}=\left(3.1 \times 10^{31} \div 1.17 \times 10^{33}\right)>10^{24}-$ much higher than the limit imposed by the eqn. $(7)$ of $\mathrm{T}_{\mathrm{BE}}(\mathrm{B}=0)$.

Because that- according to the last astrophysical data, the determined values of the "black hole" magnetic field are much lower than those predicted by the theoretical models [15], it results that the previous deduced conditions for B and $\mathrm{T}_{\mathrm{p}}$ may exists only in the outer space at enough far distance of a magnetaric star.

For example, if we have- according also to eqn. (1), a magnetaric field:

$$
\mathrm{B}(\mathrm{R})=\mathrm{k}_{1} \boldsymbol{\rho}_{\mathrm{c}} \mathrm{v}_{\mathrm{c}}=\mathrm{k}_{1} \boldsymbol{\rho}_{0}\left(\frac{\mathrm{R}_{0}}{\mathrm{R}}\right)^{2} \mathrm{c} \frac{\mathrm{R}_{0}}{\mathrm{R}}=\mathrm{B}_{0}\left(\frac{\boldsymbol{R}_{0}}{\boldsymbol{R}}\right)^{3}
$$


with $\mathrm{B}_{0} \approx 10^{11} \mathrm{~T}$ at the magnetar's surface, $\mathrm{S}_{0}\left(\mathrm{R}_{0}\right)$, it results that the values: $B=\left(2.2 \times 10^{6} \div 8.3 \times 10^{7}\right) \mathrm{T}$ are attained in the interval: $\Delta \mathrm{R}_{\mathrm{T}}=(35 \div 10,6) \mathrm{R}_{0}$.

Taking into account also the cooling effect of the magnetic field, resulted according to CGT and by the effect of the $\mu_{1}$ potential, it results that in the outer space of a magnetar, in the distance interval $\Delta \mathrm{R}_{\mathrm{T}}$, the specific condition: $\mathrm{T}_{\mathrm{p}}=$ $\left(4.8 \times 10^{-11} \div 1.8 \times 10^{-10}\right) \mathrm{K}$, of $\mathrm{P}^{\mathrm{c}}$-pre-clusters forming, may be

$$
\left(-\frac{\hbar^{2}}{2 \mathrm{~m}} \frac{\partial^{2}}{\partial r^{2}}+V(r)+V_{i}\left(a_{s}\right)\right) \psi(r)=\mu \cdot \psi(r) ;
$$

specific to temperatures much smaller than $T_{B E}$, in which: $m$ is the boson's mass, $a_{\mathrm{s}}$ is the scattering length, $\mu$ is the chemical potential and $\mathrm{N}$ is the number of bosons, the possibility of bosons confining in an etherono-quantonic vortex-tube $\xi_{\mathrm{B}}$ which materializes the magnetic field lines, can be equated by considering a negative value of the coherent scattering lengt (a) -corresponding to an attractive interaction, the external potential $\mathrm{V}(\mathrm{r})$ being- in this case, a magneto-gravitic potential $\mathrm{V}_{\mathrm{MG}}$ given by the local gradient $\nabla_{\mathrm{r}}$ $\left(\rho_{\Gamma} c\right)$ of the etherono-quantonic vortex-tubes $\xi_{B}$ that materializes the field lines of magnetic induction B, [2-4].

\section{The Gravito-Magnetic Potential of the Magnetic Field Lines}

When the total attractive potential: $\mathrm{V}_{\mathrm{a}}=-\left(\mathrm{V}_{\mathrm{MG}}+\mathrm{V}_{\mathrm{i}}\right)$ is much higher than $\mathrm{k}_{\mathrm{B}} \mathrm{T}$, we have: $\mu \approx \mu_{1}=\mathrm{V}_{\mathrm{a}}$, which is - in this case, the effective chemical potential.

If $\mathrm{V}_{\mathrm{i}}=\mu_{\gamma} \cdot \mathrm{B}$, it results that the considered magneto-gravitic

$$
\mathrm{m}_{\varphi}=\int 2 \pi r \rho_{\varphi}^{0} \frac{r_{0}}{r} d r=\pi \cdot r_{\varphi}^{2} \cdot \rho_{\varphi}=\pi \cdot r_{\varphi}^{2} \frac{B(R)}{k_{1} \cdot c}=\frac{B(R)}{k_{1} \cdot c} \frac{1}{n_{\varphi}}=\frac{\varphi_{0}}{k_{1} c}=\frac{h}{2 e \cdot k_{1} c}=4.27 \times 10^{-14} \mathrm{~kg} / \mathrm{m} ; \quad \mathrm{k}_{1}=1.56 \times 10^{-10} \frac{\mathrm{m}^{2}}{C}
$$

It results also that: $r_{\phi}=\sqrt{ }\left(m_{\phi} k_{1} c / B(R)\right)$. For example, for B $\approx 10^{6} \mathrm{~T}$ it results: $\mathrm{r}_{\phi}=4.47 \times 10^{-11} \mathrm{~m}$-corresponding- by the equality: $\mathrm{l}_{\mathrm{i}}=2 \mathrm{r}_{\phi}$, to $\mathrm{N}=1.4 \times 10^{30}$ and for $\mathrm{B}=10^{11} \mathrm{~T}$ it results: $\mathrm{r}_{\phi}=1.41 \times 10^{-13} \mathrm{~m}$-corresponding -by the equality: $\mathrm{l}_{\mathrm{i}}=2 \mathrm{r}_{\phi}$, to $\mathrm{N}=4.45 \times 10^{37}$, (compared with $\mathrm{N}_{0}=3.57 \times 10^{44}$ ). Assumingaccording to CGT [2-4], that the vortex-tubes $\xi_{\mathrm{B}}$ are formed$$
V_{i}\left(a_{s}\right)=\frac{4 \pi \cdot \hbar^{2} a_{s}}{m}|\psi|^{2} ; \quad \mathrm{N}_{\mathrm{p}}=\int|\psi|^{2} d^{3} r
$$

fulfilled.

Also, we may hypothesize that such specific conditions for $\mathrm{B}$-field and $\mathrm{T}_{\mathrm{p}}$ may be generated also by a rotational black hole with electrically charged surface, (model permitted by the general relativity).

In a Gross-Pitaevskii equation, of the BEC's wave function:

potential $\mathrm{V}_{\mathrm{MG}}$ decreases the value of the B-field at which a BEC with gammons may be obtained, resulted from the obtained equation: $\lambda_{\mathrm{t}} \sqrt{ } \mu_{1}=1.9 \times 10^{-11}$, with: $\mathrm{T}_{\mathrm{B}}=10^{3} \mathrm{~K}$ and $\mathrm{T}_{\mathrm{p}}$ $\approx \mathrm{T}_{\mathrm{h}}=4.8 \times 10^{-11} \mathrm{~K}$. For equate this effect, we may use the hypothesis of the magnetic fluxon $\phi_{0}=\mathrm{h} / 2 \mathrm{e} \approx 2 \times 10^{-15} \mathrm{~Wb}$, considering that the $\xi_{\mathrm{B}}$-vortex-tubes of the B-field are fluxon $\phi_{0}$ and that they have a linear decreasing of the impulse density: $p_{c}=\rho(r) \cdot(\omega \cdot r)=\rho(r) \cdot c$ for $r \leq r_{\phi}$ (specific to the vortex-tubes) and a mean density: $\rho_{\phi}$ approximate equal with those resulted from the local $B_{1}$-field value resulted from eqn. (1): $B_{1}=k_{1} \rho_{B} c, \rho_{B}(R)=\rho_{B}{ }^{0}\left(R_{0} / R\right)^{3}$.

For a surface $S=1 \mathrm{~m}^{2}$, the fluxons number $n_{\phi}$ rectangular on $\mathrm{S}$ are given according to the equation:

$$
\phi=\mathrm{B} \cdot \mathrm{S}=\mathrm{B} \cdot 1=\mathrm{n}_{\phi} \phi_{0} ; \Rightarrow \mathrm{n}_{\phi}=\mathrm{B} \cdot 1 / \phi_{0} ;
$$

Considering an unit length of the fluxons: $1_{\phi}=1 \mathrm{~m}$, the fluxon's mass on unit length $\mathrm{m}_{\phi}$ is given according to the relations:

$$
\mathrm{V}_{\mathrm{MG}}(\mathrm{r})=\frac{v_{1}}{2} \rho_{\varphi}(r) \cdot c^{2}=\frac{v_{1} c^{2}}{4 \pi \mathrm{r}} \sqrt{\frac{m_{\varphi} \cdot B(R)}{k_{1} \cdot c}}=\frac{v_{1} \cdot c}{4 \pi k_{1} \cdot \mathrm{r}} \sqrt{\varphi_{0} B(R)} ; \quad \rho_{\varphi}(\mathrm{r})=\rho_{\varphi}^{0} \frac{\mathrm{r}_{\mathrm{v}}}{\mathrm{r}}=\frac{m_{\varphi}}{2 \pi \cdot r_{\varphi}} \frac{1}{\mathrm{r}} ; \quad \mathrm{m}_{\varphi}=2 \pi \rho_{\varphi}^{0} r_{v} r_{\varphi} ;
$$

with:

$$
\mu_{1}=\mu_{\gamma} \cdot \mathrm{B}(\mathrm{R})+\mathrm{K}_{\mathrm{M}} \frac{\sqrt{\mathrm{B}(\mathrm{R})}}{\mathrm{r}} \quad ; \quad \mathrm{K}_{\mathrm{M}}=\frac{v_{\mathrm{i}}^{\mathrm{e}} \cdot c}{4 \pi k_{1}} \sqrt{\varphi_{0}}=7.87 \times 10^{-40}\left(\frac{\mathrm{J} \cdot \mathrm{m}}{\sqrt{\mathrm{T}}}\right) \quad ; \quad \mathrm{r}_{\varphi}=\sqrt{\frac{m_{\varphi} k_{1} \cdot c}{B(R)}}
$$

For $\mathrm{l}_{\mathrm{i}}=\mathrm{N}^{-1 / 3}=\mathrm{r}_{\mathrm{\phi}}$, we have:

$\mathrm{V}_{\mathrm{MG}}\left(\mathrm{r}_{\phi}\right)=\left(\mathrm{v}_{\mathrm{i}}^{\mathrm{e}} \cdot \mathrm{c}\right) \mathrm{B}(\mathrm{R}) / 4 \pi \mathrm{k}_{1}=1.76 \times 10^{-32} \mathrm{~B}(\mathrm{R})-$

i.e- a neglijible value comparative to:

$\mathrm{V}_{\mathrm{B}}=\mu_{\mathrm{e}} \cdot \mathrm{B} \approx 3 \times 10^{-24} \mathrm{~B}(\mathrm{R})$.

But the magneto-gravitic potential $\mathrm{V}_{\mathrm{MG}}(\mathrm{r})$ may be important for the confining of bosons with small or null magnetic moment, of the order of $10^{-31} \div 10^{-34} \mathrm{~J} / \mathrm{T}$, as those of the neutrino: $\mu_{v} \approx 10^{-10} \mu_{\mathrm{B}}$ or those of photons and may explain- in the last case, the electron's forming as collapsed Bose-Einstein condensate of photons with super-dense kernel in a magnetaric-like field [2-4].

Also, we may conclude that in the case of neutral mesons 
and baryons having small or null magnetic moment but an impenetrable quantum volume of the size order: $\left(1 \div 10^{-2}\right) \mathrm{fm}^{3}$, the potential $\mathrm{V}_{\mathrm{MG}}$ may generates dark matter bosons in a magnetaric-like field, by mesons cold confining.

For example, for the confining of $\pi$-mesons with $v_{i}^{\pi} \approx$ $2.5 \times 10^{-2} \mathrm{fm}^{3}[5]$ by a magnetaric field: $\mathrm{B} \approx 10^{11} \mathrm{~T}$, it results from eqn (13), that: $\mathrm{r}_{\phi}=1.4 \times 10^{-13} \mathrm{~m}$ and $\mathrm{V}_{\mathrm{MG}}\left(\mathrm{r}_{\phi}\right) \approx 3.8 \times 10^{-19} \mathrm{~J}$ $\approx 2.4 \mathrm{eV}$, corresponding to a confining force: $\mathrm{F}_{\mathrm{MG}}\left(\mathrm{r}_{\phi}\right)=$ $2.7 \times 10^{-6} \mathrm{~N}$.

In the same-time, it results that-by the $\mathrm{V}_{\mathrm{MG}}$ potential, the $\xi_{\mathrm{B}}$-vortex-tubes helps the pearlitization of a formed BEC into pre-clusters with $\mathrm{N}_{0^{-}}$- density of particles and relative small number of bosons and the gammonic pre-clusters' collapsing and their transforming into particles.

For example, considering a radius $r_{p}$ of meta-stable equilibrium of a drop of BEC formed by the BEC's pearlitization and maintained by the equilibrium between the force generated by the internal thermal energy $F_{t}\left(r_{p}\right)=$ $\mathrm{V} \cdot \mathrm{N}_{0} \mathrm{k}_{\mathrm{B}} \mathrm{T}_{\mathrm{i}}$ and the force generated by the surface tension, $\sigma$ :

$$
\frac{d E}{d r}=-P_{0} \frac{d V}{d r}+\sigma \frac{d S}{d r}=0 ; \mathrm{V}=\frac{4 \pi}{3} r^{3} ; \mathrm{S}=4 \pi \cdot \mathrm{r}^{2} ;
$$

because that $\sigma=(1 / 2) \mathrm{F}_{\gamma} / 1$, (the force rectangular on unit length), for: $\mathrm{N}_{0} \approx 1 / \mathrm{a}^{3}=3.57 \times 10^{44},(\mathrm{a}=1.41 \mathrm{fm}$ - the metastable equilibrium inter-distance between gammons [7]), and because the antiparallel magnetic moments of two adjacent gammons of a precluster have a residual (reciprocal) value given by: $\mathrm{E}_{\gamma}=2 \mathrm{~m}_{\mathrm{e}} \mathrm{c}^{2} \approx \mathrm{V}_{\mathrm{e}}\left(\mathrm{a}, \mathrm{e}^{*}\right)+\mathrm{V}_{\mu}^{\mathrm{e}}\left(\mathrm{a}, \mathrm{e}^{*}\right) \approx 3 \mathrm{~V}_{\mu}^{\mathrm{e}}\left(\mathrm{a}, \mathrm{e}^{*}\right)$ and: $\mathrm{B}(\mathrm{d}) \approx \mathrm{E}(\mathrm{d}) / \mathrm{c},[7]$, giving: $\left.\mathrm{e}^{*} \approx \sqrt{(}{ }^{2} / 3\right) \cdot \mathrm{e}$, it results that:

$$
\mu_{1}(\mathrm{a})=\frac{\boldsymbol{m}_{e}}{\boldsymbol{m}_{\boldsymbol{\gamma}}} \frac{\mathrm{e}^{*} \boldsymbol{c} \cdot \boldsymbol{d}}{2}=\mu_{\mathrm{SP}} \sqrt{\frac{2}{3}} \cdot \frac{\boldsymbol{d}}{2 \cdot r_{\lambda}} ; \mathrm{r}_{\lambda}=\frac{\hbar}{\mathrm{m}_{\mathrm{c}} \boldsymbol{c}} ; \mathrm{e}^{*}=\frac{4 \pi(0.9 \mathrm{~d})^{2}}{\boldsymbol{k}_{1}}=\sqrt{\frac{2}{3}} \boldsymbol{e} ; \mathrm{d}=\mathrm{a}
$$

and-by CGT, because $\mathrm{m}_{\mathrm{e}} \mathrm{c}^{2}=\mathrm{e}^{2} / 8 \pi \varepsilon_{0} \mathrm{a}$, it results that:

$$
V_{\mu}^{e}(a)=-\frac{e^{*}}{8 \pi \varepsilon_{0} a}=-\left(\frac{2}{3}\right) \frac{e^{2}}{8 \pi \varepsilon_{0} a} ; \Rightarrow \frac{F_{\gamma}}{1} \approx \frac{F_{\mu}^{e}}{a}=\frac{\mu_{0}}{2 \pi} \frac{2}{3} \frac{\mu_{B P}^{2}}{r_{\lambda}^{2} a^{3}}=\frac{e^{2}}{12 \pi \varepsilon_{0} a^{3}}
$$

and because the electric force between gammons may be neglected, the meta-stable equilibrium radius has the form:

$$
r_{p}=\frac{2 \sigma}{P_{0}}=\frac{F_{\gamma}}{l \cdot P_{0}} \approx \frac{\mu_{0}}{3 \pi} \frac{\mu_{B P}^{2}}{r_{\lambda}^{2} a^{3}} \frac{1}{N_{0} k_{B} T_{i}}=\frac{\mu_{0}}{3 \pi} \frac{\mu_{B P}^{2}}{r_{\lambda}^{2}} \frac{1}{k_{B} T_{B}} \approx 5.5 x \frac{10^{-6}}{T_{B}}[\mathrm{~m}]
$$

For $\mathrm{T}_{\mathrm{B}} \approx 10^{3} \mathrm{~K}$ it results: $\mathrm{r}_{\mathrm{p}} \approx 5 \times 10^{-9} \mathrm{~m}$, so the pearlitization with the forming of quasi-cylindrical pre-clusters of baryonic neutral particles corresponding to a radius: $r_{b}<r_{a}$ may be formed by large oscillations of the internal temperature $T_{i}$ given by the zeroth vibrations, around the value $T=T_{B}$.

If we consider a BEC of $z^{0}$-preons- formed by clusterizing and collapsing process as neutral couple of two quarcins $\left(\mathrm{m}_{\mathrm{q}}\right.$ $\approx 17 \mathrm{~m}_{\mathrm{e}}$ ) with degenerate charge $\mathrm{e}^{*}$, axially coupled, with the same value $\mathrm{N}_{0}$ and with $\mathrm{T}_{\mathrm{B}} \approx 86 \mathrm{~K}$-given by (8), it results from eqns. (17)-(19), that: $\mu_{\mathrm{z}} \approx \mu_{\gamma} / 17$ and: $\mathrm{r}_{\mathrm{p}} \approx 4 \times 10^{-11} \mathrm{~m}$.

Under this value $r_{p}$ of metastable equilibrium, because the decreasing of the internal energy, the residual (reciprocal) magnetic moments of the gammons generates the precluster's collapsing, without destruction- conform to CGT.
If the internal pre-cluster's temperature $T_{i}$ is maintained close to the metastable equilibrium value $T_{B}$, the pre-cluster's collapsing may still occur in a strong magnetic field, by the aid of the magneto-gravitic potential $\mathrm{V}_{\mathrm{MG}}\left(\mathrm{r}_{\phi}\right)$, according to the model.

So, it is argued the conclusion that also a static but strong magnetic field may create conditions for BEC forming andparticularly, for particle-like collapsed BEC forming, by bosons capturing with the $\xi_{\mathrm{B}}$-vortex-tubes which materializes the B-field lines.
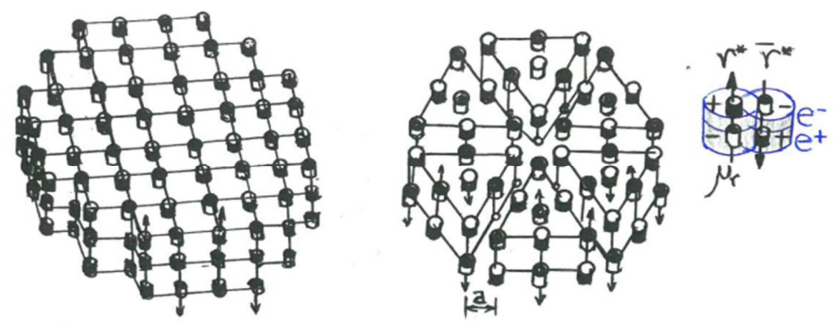

Figure 1. Part of a crystallized gammonic pre-cluster which may result by a BEC's pearlitizing, (CGT).

It results-in consequence, two plausible scenario of particles cold genesis-according to CGT: a) by clusterization, from $\mathrm{z}^{0^{*}}$ - preonic pre-clusters, in a step-by step scenario, by steps of $\mathrm{z}^{*}-\mathrm{q}^{0^{*}}$ - pre-clusters cold collapsing, or: b) by the pearlitization of a bigger BEC, by the temperature oscillation around the value $T_{B}$, with the cold collapsing of the resulted BEC pre-cluster but without destruction.

Relative to the particles genesis from quantum vacuum fluctuations, considered in SVT but also in CGT- as chiral (vortexial) fluctuations, contrary to the scenario of spontaneous particles-antiparticles pairs forming from virtual particle-like pairs, supposed by the Heisemberg's nedetermination relation of quantum mechanics, it results from the previous analyze, that the fermions and bosons forming process is possible only by enough strong vortexial fluctuations in the etherono-quantonic quantum vacuumwhich determine also the speed of the process and the mass of the formed particles, resulting as more plausible a "stepby-step" scenario of particles forming by chiral fluctuations, beginning with the vectorial photons forming- process possible also at lower density of the quantum vacuum, comparable with those of a lower magnetic field: $\rho_{B}(B) \approx$ $\rho\left(m_{p}\right)$.

For example, for the cold genesis of a quantum of $2.7 \mathrm{~K}$ radiation, if it is formed from two vectorial photons named "vectons" in CGT, with mass: $\mathrm{m}_{\mathrm{v}}=2.3 \times 10^{-40} \mathrm{~kg}$ and considered with an inertial mass of gauge radius: $\mathrm{r}_{\mathrm{v}}=0.41 \mathrm{a}=$ $0.6 \mathrm{fm}-$ in the free state, it results that: $\rho_{\mathrm{B}}(\mathrm{B}) \approx \rho\left(\mathrm{m}_{\mathrm{v}}\right)=$ $2.5 \times 10^{5} \mathrm{~kg} / \mathrm{m}^{3}$, resulting that a vortexial magnetic-like field corresponding to $\mathrm{B}_{\mathrm{v}}=\mathrm{k}_{1} \rho_{\mathrm{B}} \mathrm{c} \approx 10^{4} \mathrm{~T}$ may generates vectons from the primordial dark energy- composed of etherons and quantons $\left(m_{h} c^{2}=h \cdot 1\right)$, according to CGT, the cold genesis of electrons being possible at higher values of magnetic-like energy density, in a magnetaric-like field, $[9,10]$.

It results- according to CGT, that the forming of virtual particles from the energy of quantum vacuum by chiral 
fluctuation and their transforming into real particles involve either a magnetic-like (vortexial) field of values higher than a critical value: $\mathrm{B}_{\mathrm{v}} \approx 10^{4} \mathrm{~T}$, or already formed gammonic pairs $\gamma^{*}\left(\mathrm{e}^{-} \mathrm{e}^{+}\right)$and a low temperature: $\mathrm{T}<<10^{3} \mathrm{~K}$, and because that the relative stability of its specific structure is ensured only by the existence of superdense kernels (centroids), resulted from confined quantons- in CGT, which stabilizes the formed vortex(es) and its vortexial structure.

The explaining of the strong interaction with mass excess, in which the total mass of resulted particles exceed the total mass of particles entered in reaction, results as in the case of reaction:

$\pi^{-}\left(\overline{\mathrm{m}}_{1}+\mathrm{m}_{2}\right)+\mathrm{p}_{\mathrm{r}}\left(2 \mathrm{p}^{+}+\mathrm{n}^{-}\right)+\mathrm{Q} \rightarrow \Lambda^{\mathrm{o}}(\mathrm{s}+\mathrm{n}+\mathrm{p})+\mathrm{K}^{\mathrm{o}}\left(\mathrm{m}_{2}+\bar{\lambda}\right)$, by the participation of real bosons of quantum vacuum, particularly-dark matter bosons, formed by quark-antiquark pairs, with the intrinsic energy $m_{b} c^{2}$ lower than the interaction energy, $Q_{i},[3,4,7]$.

For a Bose-Einstein condensate in a gravitational potential $\mathrm{V}_{\mathrm{g}}=\mathrm{m} \cdot \mathrm{g} \cdot \mathrm{y}$, because that the transition temperature is increased by the presence of the gravitational field [9], for the forming of a gammonic pre-cluster with a density of gammons: $\mathrm{N}_{0} \approx 1 / \mathrm{a}^{3}=3.57 \times 10^{44}$ we may conclude that in the gravitational field of a black hole with a surface temperature $\mathrm{T}_{\mathrm{t}}<\mathrm{T}_{\mathrm{B}}(\mathrm{g}=0)$ given by eqn. (7), the conditions of the $\mathrm{P}^{\gamma}$-precluster forming are fulfilled if it is obtained a specific gammons concentration, for example: $\mathrm{N} \geq 10^{24}$, for $\mathrm{T}_{\mathrm{B}} \approx$ $1464 \mathrm{~K}$. A concentration $\mathrm{N}>10^{24}$ of gammons may results by gammons gravitational attraction toward the black hole's surface, the condition: $\mathrm{T}_{\mathrm{p}}<\mathrm{T}_{\mathrm{BE}}$ at the BH's surface being -in this case, fulfilled, because the very low temperature at the BH's surface.

We may consider also a neutrino magnetic moment: $\mu_{v} \approx$ $10^{-10} \mu_{\mathrm{B}}$ corresponding to the superior limit deduced by SuperKamiokande collaboration [16], and a neutrino mass used by CGT: $\mathrm{m}_{\mathrm{v}} \approx 10^{-4} \mathrm{~m}_{\mathrm{e}}=9 \times 10^{-35} \mathrm{~kg}$, (comparative with those indicated by recent experimental data [17]: $\mathrm{m}_{\mathrm{v}} \approx$ $1.85 \mathrm{eV}=3.3 \times 10^{-36} \mathrm{~kg}$ ), which corresponds in CGT to a couple of antiparallel electronic centroids with antiparallel chiralities and a radius of its section: $\mathrm{r}_{0} \approx 10^{-18} \mathrm{~m}$.

A Bose-Einstein condensate of neutrinos may be considered as cluster of gammonic superdense centroids confined in a volume comparable with those of a constituent quark mass, (considered in CGT with $\sim 378$ gammons with the centroids in a volume $v_{\mathrm{q}}\left(\mathrm{r}_{\mathrm{n}} \approx 1 \mathrm{fm}\right)$, with an approximate density of particles: $\mathrm{N}_{0}{ }^{\mathrm{v}} \approx 10^{47}$ which- in a magnetic B-field, gives- by eqn. $(6)$, a value $\mathrm{L} \approx 4.6 \times 10^{-14} \mathrm{~m}$.

For the situation of a neutrinos flux generated by a star situated in the vicinity of a Black hole, of value comparable with those of the solar neutrinos at the Earth's surface: $\phi_{v}=$ $\mathrm{n}_{0} \cdot \mathrm{c} \approx 6.5 \times 10^{14} / \mathrm{m} \cdot \mathrm{s}$, we have: $\mathrm{N}=\mathrm{n}_{0}$, resulting- by eqn. (7), that temperatures $\mathrm{T}<\mathrm{T}_{\mathrm{B}} \approx 6 \times 10^{-5} \mathrm{~K}$ as those considered for the surface of a black hole star, may generates the forming of Bose -Einstein condensate of neutrinos, which- at higher dimensions than those of an atomic nucleus, may initiates the cold forming of a micro-black-hole.

If -instead of antiparallel coupled electronic centroids forming electronic neutrinos we have electronic centroids with antiparallel chiralities axially coupled, it results in consequence -according to the cold genesis quasi-crystallin quark model of CGT, the possibility of particles forming starting with the forming of a Bose-Einstein condensate of electronic centroids with antiparallel chiralities axially and parallely coupled (on axial, respective-on radial direction), which- after the pearlitization of the BEC, may forms mesonic or baryonic quasi-crystallin kernels which initiates the forming of etherono-quantonic vortexes around their center, by the action of quantum and sub-quantum winds, corresponding to magnetic moments which attracts- by the resulted self-potential $\mathrm{V}=\mathrm{V}_{0}|\psi|^{2}$, some photons of the quantum vacuum, (previously formed), which- in this way, generates the quantum volume of the resulted composite particle, according to CGT.

The previous conclusions argues the possibility of dark matter bosons genesis in the field of a black hole or of a magnetar-type star- at an enough long distance from its surface.

\section{Conclusions}

In the present paper, based on a previous work [7] regarding the cold particles forming process as collapsed cold cluster of gammons- considered as gammonic pairs: $\gamma^{*}=\left(\mathrm{e}^{-} \mathrm{e}^{+}\right)$of axially coupled electrons with opposed charges, we analyze the possibility of gammons pre-cluster forming, specific to cold formed quarks or pseudo-quarks (corresponding to neutral bosons), as Bose-Einstein condensate formed in the magnetic and in the gravitational field of a star which ensures at its surface the condition: $\mathrm{T}<\mathrm{T}_{\mathrm{BE}}$.

By known relations of a BEC forming, it is argued that- in the magnetic field of a star, the forming of a gammonic BoseEinstein condensate with particles density $\mathrm{N}_{0}$ corresponding to those of a pre-cluster of gammons which may generates a particle-like stable cluster, may occurs- for a transition temperature $\mathrm{T}_{\mathrm{BE}} \approx 10^{3} \mathrm{~K}$, in an specific interval of field intensity and of temperature: $\mathrm{B}=\left(2.2 \times 10^{6} \div 8.3 \times 10^{7}\right) \mathrm{T}$ and $\mathrm{T}_{\mathrm{p}}=\left(4.8 \times 10^{-11} \div 1.8 \times 10^{-10}\right) \mathrm{K}$, which may exists in the surrounding vacuum of a magnetar-type star or of a rotational black hole with electrically charged surface, which may generate also a strong magnetic field.

The possible mechanism of the formed BEC transforming into pre-clusters of gammons which may become particlelike collapsed BEC, is a pearlitization mechanism, resulted as fragmentation of the formed BEC.

It is argued also that in the gravitational field of a black hole with a surface temperature $\mathrm{T}_{\mathrm{t}}<\mathrm{T}_{\mathrm{BE}}(\mathrm{g}=0)$, the conditions of gammonic pre-clusters forming are fulfilled if it is obtained a specific gammons concentration which may results by gammons gravitational attraction toward the black hole's surface.

Also, it is argued that temperatures $\mathrm{T}<\mathrm{T}_{\mathrm{B}}$ as those considered for the surface of a black hole star, may generates the forming of Bose -Einstein condensates of neutrinos, which- at higher dimensions than those of an atomic nucleus, may initiates the cold forming of dark matter bosons or of a 
micro-black-hole.

In the same time, this possibility sustains- by the proposed model of particles forming, the possibility of dark superheavy particles/bosons existence- supposed by some dark matter models, as particle-like collapsed Bose-Einstein condensate formed in a strong magnetic or/and gravitational field, with a quasi-crystallin arrangement at the particle kernel's level, obtained as consequence of the very low temperature of the formed particle, with the aid of the field, and maintained by the generating of a repulsive potential $\mathrm{V}_{\mathrm{r}}(\mathrm{d})$ of short range, by the 'zeroth' vibrations of the superdense electronic centroids $\left(\sim 10^{-18} \mathrm{~m}\right.$ diameter $)$ which determines local destruction of internal vortexial structures (cold photons), releasing their energy in form of quantum flux which impede the particle's kernel collapse.

It is argued that the particles forming from quantum vacuum fluctuations- considered in the quantum mechanics, is possible at $\mathrm{T} \rightarrow 0 \mathrm{~K}$, either by a vortexial, magnetic-like field, corresponding to $\mathrm{B} \geq 10^{4} \mathrm{~T}$, with the producing of dense kernel(s) which may stabilize the quantum vortex, or by already formed gammons, in a "step-by-step" process, by clusterizing or pearlitizing and the cold collapsing of the resulted pre-clusters, without their destruction.

The possibility to explain the masses and the magnetic and electric properties of the elementary particles resulted from the cosmic radiation in a preonic model, by a cold clusterizing process and with only two quasi-crystallin basic bosons: $\mathrm{z}_{2}=4 \mathrm{z}^{0}=136 \mathrm{~m}_{\mathrm{e}} ; \mathrm{z}_{\pi}=7 \mathrm{z}^{0}=238 \mathrm{~m}_{\mathrm{e}}$, indicates -in our opinion, that- after the electrons (negatrons and positrons) forming, the clusterizing was the main process of the particles forming in the Universe, by at least two steps: a)- the quasi-crystallin pre-cluster forming (of gammons or of formed $\mathrm{z}^{0}$-preons or $\mathrm{z}_{2}$ - and $\mathrm{z}_{\pi^{-}}$zerons) and $\mathrm{b}$ ) -the precluster's cold collapsing, without destruction, with the maintaining of a quasi-crystallin arrangement of electronic centroids at the kernel's level.

The resulted explicative model of particles cold genesis may explain the existence of a huge number of material particles in the Universe by the conclusion of cold ("dark") photons and thereafter- of electronic neutrinos and cold electrons genesis in the Cold Proto-Universe's period, by chiral (vortexial) fluctuations in the 'primordial dark energy'considered in CGT as omnidirectional fluxes of etherons and quantons circulated through a brownian part of etherons and quantons.

The generalization to the scale of an atomic nucleus permits to consider an atomic nucleus as a (non-collapsed) fermionic condensate with quasi-crystallin arrangement of nucleons, which may explain the nucleonic "magic" numbers of maximal stability [2-4], the nuclear fission reactions- well described by the droplet nuclear model, being explained by a nuclear local phase transformation at the internal temperature increasing -determined by the nucleons' vibrations.

\section{References}

[1] Sinha, K. P., Sudarshan, E. C. G., „The superfluid as a source of all interactions", Found. of Physics, Vol. 8, Issue 11-12, Dec. (1978), pp. 823-831.

[2] Arghirescu, M., "The Cold Genesis", Ed. Invel- Multimedia, 2011; viXra, 1104. 0043, (2012).

[3] Arghirescu, M., "The Cold Genesis of Matter and Fields", Ed. SciencePG, (2015).

[4] Arghirescu, M., 'A Quasi-Unitary Pre-Quantum Theory of Particles and Fields and Some Theoretical Implications', IJHEP, July, 80-103, (2015).

[5] Anderson, C. D., 'The Positive Electron', Phys. Rev. 43, 491, 15 March (1933).

[6] Arghirescu, M., 'A preonic quasi-crystal quark model based on a cold genesis theory and on the experimentally evidenced neutral boson of $34 \mathrm{~m}_{e}$, Global Journal of Physics Vol. 5, No 1, (2016), pp. 496-504.

[7] Arghirescu M., 'The Explaining of the Elementary Particles Cold Genesis by a Preonic Quasi-Crystal Model of Quarks and a Pre-Quantum Theory of Fields', IJHEP, Vol. 5, No. 1, (2018), pp. 12-22.

[8] Perez Rojas H., Villegas-Lelovski L., 'Bose-Einstein Condensation in a Constant Magnetic Field', Brazilian Journ. of Phys., vol. 30, no. 2, June, (2000), pp. 410-418.

[9] J. I. Rivas, A. Camacho, 'Bose-Einstein condensates in a homogeneous gravitational field', arXiv: 1101. 1263v1 [condmat. quant-gas] 6 Jan (2011).

[10] Elizabeth A. Donley, N. R., Claussen, S. L. et al. 'Dynamics of collapsing and exploding Bose-Einstein condensates', arXiv: cond-mat/0105019v3, June (2001).

[11] Claussen N. R., Kokkelmans S. J. J. M. F., Thompson S. T., Donley E. A., Hodby, E., Wieman C. E. Phys. Rev. A 67 060701(R), (2003).

[12] Böhmer C. G., Harko T., 'Can dark matter be a Bose-Einstein condensate?’, arXiv:0705.4158v4 [astro-ph] 21 Jun (2007)

[13] Alfaro J., Espriu D., Gabbanelli L., 'Bose-Einstein graviton condensate in a Schwarzschild black hole', IOP Journal, Vol. 35, No. 1, Nov. (2017); arXiv: 1609. 01639v1 [gr-qc] 6, Sept. (2016).

[14] A. D. Linde, Phys. Lett. B 86, 39 (1979).

[15] Dallilar Y., Eikenberry S. S., Garner A., 'A precise measurement of the magnetic field in the corona of the black hole binary V404 Cygni', Science Vol. 358, Issue 6368, 08 Dec. (2017), pp. 1299-1302

[16] D.W.Liu et al., [Super Kamiokande Collaboration], Phys.Rev.Lett.93, 021802(2004); arXiv: hep-ex/0402015.

[17] Nieuwenhuizen, T. M., 'Dirac neutrino mass from a neutrino dark matter model for the galaxy cluster Abell 1689', Journal of Physics: Conference Series, 701 (1): 012022, (2016); arXiv: 1510.06958. 